\title{
How to Focus a Bose-Einstein Condensate in a Waveguide
}

\section{A new "lensing" technique counters the spreading of an ultracold cloud of atoms inside a tiny waveguide.}

\section{By David Ehrenstein}

$\Lambda^{2}$ $\mathrm{n}$ ultracold cloud of atoms spreads with time, just as a beam of light spreads in space, but an electromagnetic "lens" can "focus" the cloud and reduce its kinetic energy. The lowest energies yet achieved-in the picokelvin (pK) range-require large facilities that allow the atoms to fall freely for several seconds. Now researchers have demonstrated a lens that works in this regime with atoms in a ring-shaped waveguide less than a millimeter across [1]. Manipulating these "matter waves" in small spaces is essential for exploiting their quantum effects for purposes such as high-precision navigation.

The team, led by Wolf von Klitzing at the Institute for Electronic Structure and Lasers, part of the Foundation for Research and Technology - Hellas in Greece, placed a

several-hundred-micrometer-wide Bose-Einstein condensate (BEC) of rubidium atoms into a ring-shaped electromagnetic trap, somewhat like placing a marble in a roulette wheel. To start the "marble" moving, the team tilted the ring toward the

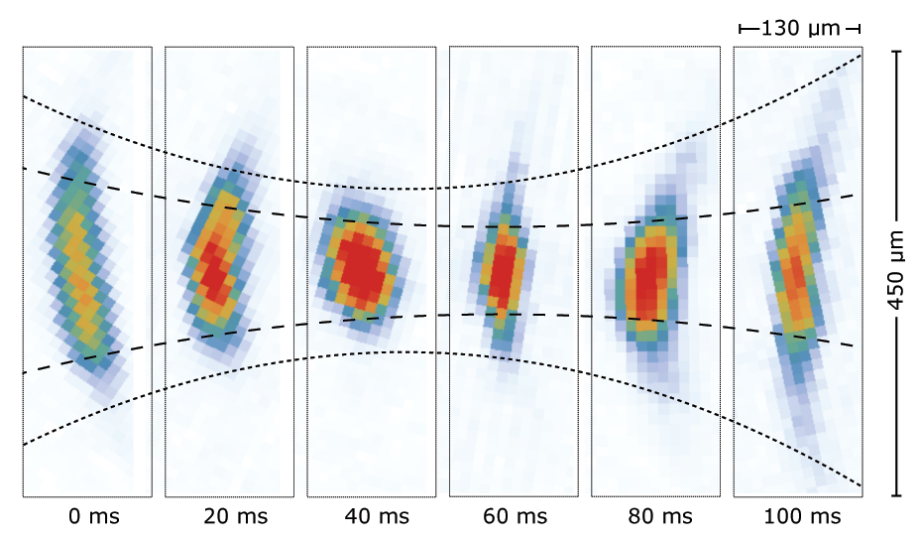

Credit: S. Pandey et al. [1] atoms and then rotated it about its original vertical axis, so that the lowest point revolved at up to $10 \mathrm{~Hz}$ for about $200 \mathrm{~ms}$. Next, they leveled the ring and let the cloud spread freely as it raced around. Finally, the team focused the BEC by again tilting the rotating ring toward the atoms for $17 \mathrm{~ms}$.

The researchers showed that the tilt angle was analogous to the focusing strength of an optical lens. Too large a tilt caused the cloud to briefly shrink and then enlarge, like an overly powerful lens focusing light in front of the intended focal plane. The ideal tilt of about $5^{\circ}$ held the cloud at a fixed size for nearly a second and reduced its expansion energy to $800 \mathrm{pK}$.

David Ehrenstein is a Senior Editor for Physics.

\section{REFERENCES}

1. S. Pandey et al., "Atomtronic matter-wave lensing," Phys. Rev. Lett. 126, 170402 (2021). 